\title{
The Use of Pre and Post Processing to Enhance Mandible Segmentation using Active Contours on Dental Panoramic Radiography Images
}

\author{
${ }^{1}$ Nur Nafi'iyah \\ Departement of Informatics \\ Institut Teknologi Sepuluh Nopember \\ Surabaya, Indonesia \\ nurnafiiyah.19051@mhs.its.ac.id \\ ${ }^{4}$ Eha Renwi Astuti \\ Faculty of Dental Medicine \\ Universitas Airlangga \\ Surabaya, Indonesia \\ eha-r-a@fkg.unair.ac.id
}

\author{
${ }^{2}$ Chastine Fatichah \\ Departement of Informatics \\ Institut Teknologi Sepuluh Nopember \\ Surabaya, Indonesia \\ chastine@if.its.ac.id \\ ${ }^{3}$ Darlis Herumurti \\ Departement of Informatics \\ Institut Teknologi Sepuluh Nopember \\ Surabaya, Indonesia \\ darlis@if.its.ac.id
}

\begin{abstract}
Mandibular segmentation is indispensable to support the automation of the gender detection system based on the dental panoramic radiography image. However, the dental panoramic radiography image has low image contrast, the gray intensity value inhomogeneous, and the gray intensity value between the teeth and mandibular bone is almost indistinguishable. So, a good segmentation method is required to separate the mandible and teeth properly. This study aims to analyze the effect of the use of preprocessing and post-processing to enhance mandible segmentation on dental panoramic radiography images properly. In the preprocessing, we use contrast enhancement and Gaussian filters to make the mandibular area more prominent. Meanwhile, in the post-processing, we use erosion and opening morphology to remove the tooth area attached to the mandible. The mandibular segmentation uses the Active Contours method with predefined contour initialization. The dataset used is 86 dental panoramic radiographic images and the segmentation evaluation method uses Jaccard similarity. The experimental results show that the mandibular segmentation with preprocessing and postprocessing obtain Jaccard similarity values are 0.31 and 0.34, on average. Meanwhile, the results of mandibular segmentation with post-processing achieve the Jaccard similarity values are 0.51 and 0.52 , on average.
\end{abstract}

Keywords- dental panoramic radiography, mandible segmentation, active contour, preprocessing, post-processing

\section{INTRODUCTION}

Dental radiology techniques can be used to help identify individuals, by determining gender, age, and ethnic group [1]. Forensic odontology by measuring the ramus of the mandible flexure can be used to identify gender [2]. Individual identification including gender and age based on dental panoramic images is required for victims whose faces and fingerprints cannot be recognized, such as victims of natural disasters such as fires, bomb explosions, and so on [3].

Therefore we are interested in developing an application that can identify gender and age based on mandibular dental panoramic images. To develop this application, an image improvement process and mandibular segmentation are needed. Tooth segmentation in panoramic radiographic images is a difficult problem because the images have the same grayish intensity value between tooth elements and bone. Segmentation is a very important stage in diagnosing a computer-assisted disease. If the results of the segmentation are accurate, the results of diagnosing disease are also more accurate automatically. Therefore, it is necessary to develop a segmentation method, in this case, the mandibular segmentation method.

The mandible is the mandible, which is the largest and strongest facial bone [4]. The mandible serves as a place for the growth of lower teeth and contains blood vessels and nerves. Research related to mandibular segmentation in Panoramic X-ray is presented in [5]. They conducted mandibular segmentation using Active Contours. The results stated that the segmentation process of the tooth anatomy structure could be used for the extraction of the contours of the mandibular ramus. The segmentation process is divided into 4 subareas: superior alveolar border, mandibular lower border, ramus outer border, and mandibular process boundary. The image enhancement uses noise removal, median filter, and CLAHE. The next stage divides the upper and lower jaw with a strip. The inferior stage performs the process of detecting the mandible by detecting horizontal and vertical Canny filters, and Gaussian twice, followed by the detection of horizontal Prewitt with $3 \times 3$ kernels. Exterior stage by tracing contours (Active Contour) [5]. Other studies related to the segmentation of teeth and mandibular bone in CBCT images are segmenting and producing parts of the mandible using a deep learning algorithm [6] and histogram thresholding and polynomial fitting [7].

Active Contour is a contour-based segmentation method that has been widely used for segmenting medical images. Research [8] attempted to develop an Active Contour method based on the level set function area for lung segmentation. Active Contours can be modified by combining geometric and statistical methods. The Casselle Level set is added with a learning phase which is used for automatic initialization [9]. Also, medical images need to be segmented to display detailed information on the anatomy and structure of organs [10]. Various segmentation methods are proposed to produce objects according to the target, for example, the Active Contour Model (ACM) [11]. The Active Contour method does not have very good segmentation results, so a semi-automatic method was developed. The semi-automatic method 
consists of a binarization step, then determining the area of points for the initial initialization, and the final stage making a set of contours to improve the segmentation results [12]. Active contours often referred to as snakes, are an efficient method for segmenting medical ultrasound images. The contour active method is not accurate if the initial initialization is incorrect (far from the target limit). So, the literature [13] proposed a new initialization method based on image fusion. Segmentation of a pair of images together is called co-segmentation. Co-segmentation can be done with a dual Active Contour model. Both contours of the object are initialized simultaneously to produce segmentation according to the target object [14]. Other research states that the Active Contour model without initialization can still be used for segmentation. Initialization can be replaced by aggregating edge direction information based on the Cumani operation. The results of these studies can extract image contours precisely and are much better and faster [15]. The Active Contour model develops according to the intrinsic geometrical image. The approach is based on the relationship between Active Contours and geodesic calculations or minimum distance curves. The geodesic approach to segmenting objects based on minimum energy and geometric Active Contours is based on curve evolution [16]. An automatic segmentation based on local entropy and Active Contour models is proposed in [17]. Active Contour model based on the new area [17].

From the above explanation, panoramic radiographic images can be used as a tool for computer-based gender and age identification. The radiographic image is first segmented into the mandible, then identified parts of the mandible. The radiographic image is very difficult to segment using a simple method because the value of the gray image intensity between the teeth and the mandible is the same and almost indistinguishable. So, it needs an automatic segmentation process with the Active Contour method that performs contrast improvement, Gaussian filters, and morphological operations.

Based on previous studies related to the use of Active Contour for medical image segmentation, this study uses the Active Contour method for mandibular segmentation in dental panoramic radiographic images. However, the dental panoramic radiographic image has poor quality, namely low image contrast, the gray intensity value is not homogeneous, and the gray intensity value between the teeth and mandibular bone is almost indistinguishable. So we need a good segmentation method to properly separate the mandible and teeth. This study aims to analyze the effect of the use of preprocessing and post-processing to properly segment the mandible on panoramic dental panoramic radiographic images.

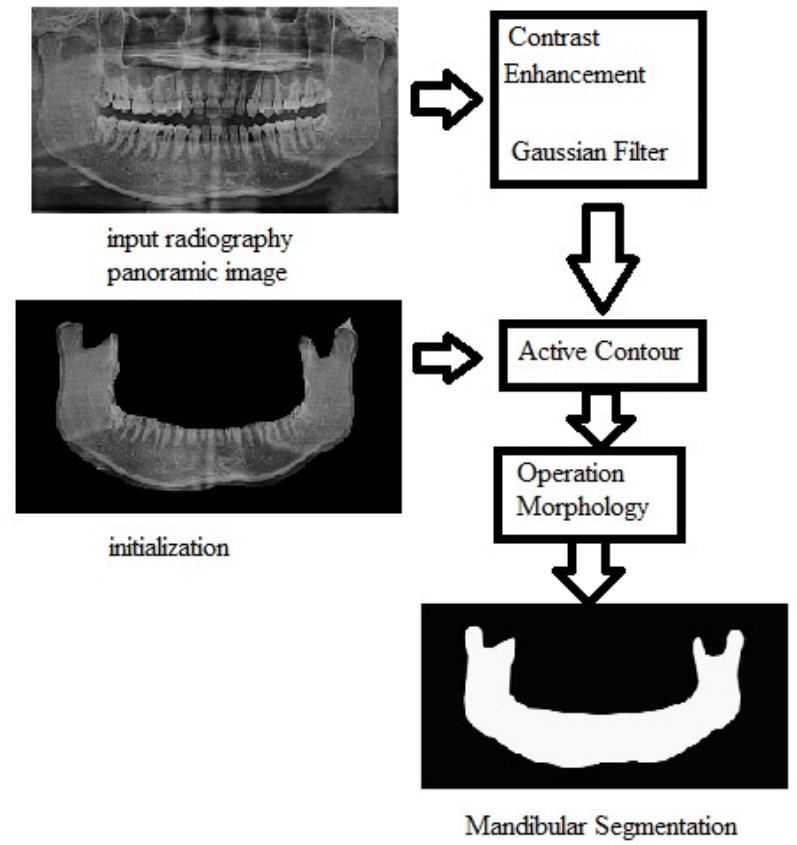

Fig. 1. The Block Diagram of Proposed Method

\section{Proposed Method}

The block diagram of the panoramic radiographic mandibular segmentation process is shown in Figure 1. The panoramic radiographic image was cropped as an Active Contour initialization image is shown in Figure 2. Panoramic radiographic images were preprocessed with contrast enhancement and Gaussian filters. The results of image preprocessing are segmented using Active Contour. The results of the Active Contour segmentation were carried out post-processing using erosion and opening morphology.

The sample of this study is secondary data in the form of soft panoramic radiographic image files from the Dental Radiology clinic of the Dental and Oral Hospital of Airlangga University, Surabaya, with good quality and confirmed by a radiologist. The sample size is determined based on the Slovin formula [18]. The error used is $10 \%$, with the hope that this research will produce a confidence value of $90 \%$. And secondary data of panoramic radiograph images with good quality were 620 . In this study, the sample size used was 86 panoramic radiographic images. The sample data has been tested like ethics by the Health Research Ethics Eligibility Commission (KKEPK) Faculty of Dentistry, Airlangga University, Surabaya with certificate number 486/HRECC.FODM/X/2020.

$$
\text { Sample }=\frac{620}{1+\left(620.10^{2}\right)}=86
$$

\section{A. Dataset}

The number of datasets used in this study was 86 panoramic radiographic images. Image resized with size $256 \times 256$ and manifold grey level, as in Figure 3 . The mandible is the lower jawbone of the face [19]. 


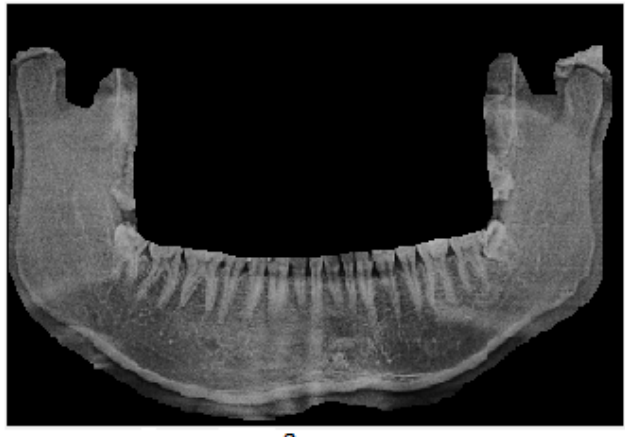

a

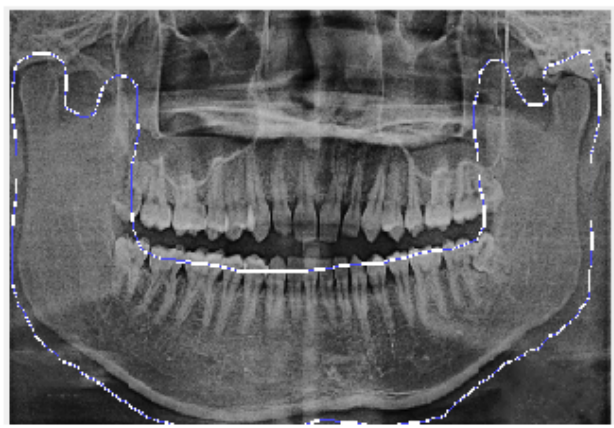

$\mathrm{b}$

Fig. 2. a. Original Image b. Active Contour Initialization

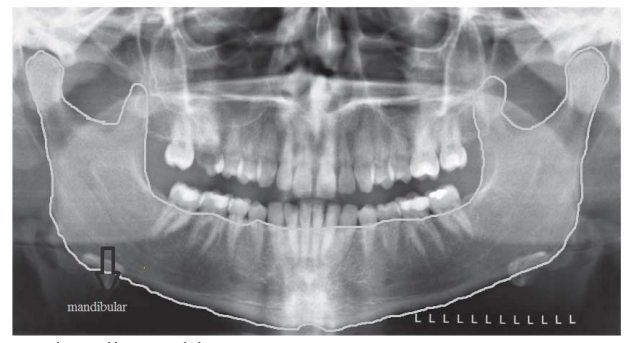

Fig. 3. Dental Radiographic Image

\section{B. Preprocessing}

\section{a. Contrast Enhancement}

The contrast in the image is the distribution of dark and light colors. An image is said to have low contrast if the color distribution is at a narrow gray level. Conversely, an image is said to have high contrast if the color distribution at the gray level is wider. Contrast is measured based on the difference between the highest and lowest intensity values that make up each image pixel. Low contrast images occur in poor or unequal lighting conditions. This is due to the non-linear image capture sensor. The distribution of pixel intensity can be stretched in contrast with Equation 1.

$$
g(x, y)=\alpha \cdot f(x, y)
$$

Based on Equation 1, the contrast will increase if $\alpha>1$, and the contrast will decrease if $\alpha<1$. Consider Figure 4 a panoramic radiographic image stretched in contrast with a value of $\alpha=2.5$. Figure 5 is a panoramic radiographic image that is stretched in contrast with a value of $\alpha=0.8$, the results do not change much from the original image.

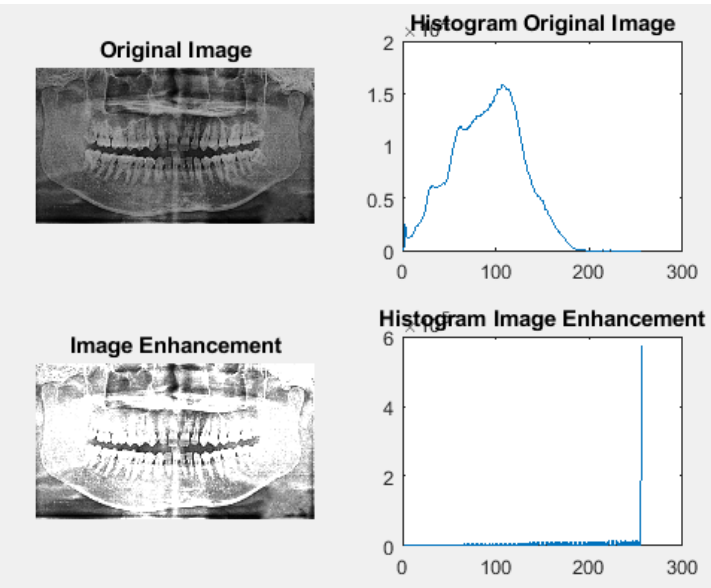

Fig. 4. Stretch Contrast $\alpha=2.5$

Image enhancement can use brightness and contrast stretching. This operation can be combined with Equation 2 . From the image improvement integration algorithm in Equation 2, the results are tested as shown in Figure 6 with the values $\beta=-45$ and $\alpha=11$. In Figure 7, the results of the repair trial by combining brightness and contrast stretching, the values of $\beta=45$, and $\alpha=1.5$.

$$
g(x, y)=(\alpha \cdot f(x, y))+\beta
$$
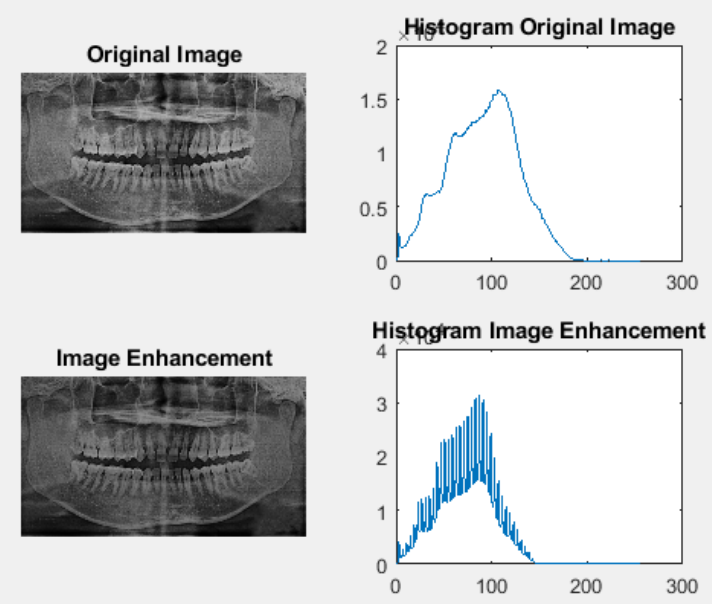

Fig. 5. Stretch Contrast $\alpha=0.8$

\section{b. Gaussian Filter}

Gaussian filters use a Gaussian function to smooth 2D images. Equation 3 is a Gaussian function.

$$
g(x, y)=e^{\frac{x^{2}+y^{2}}{2 \sigma^{2}}}
$$

Where $x, y$ is the center point of the image and $\sigma$ is the image standard deviation. 


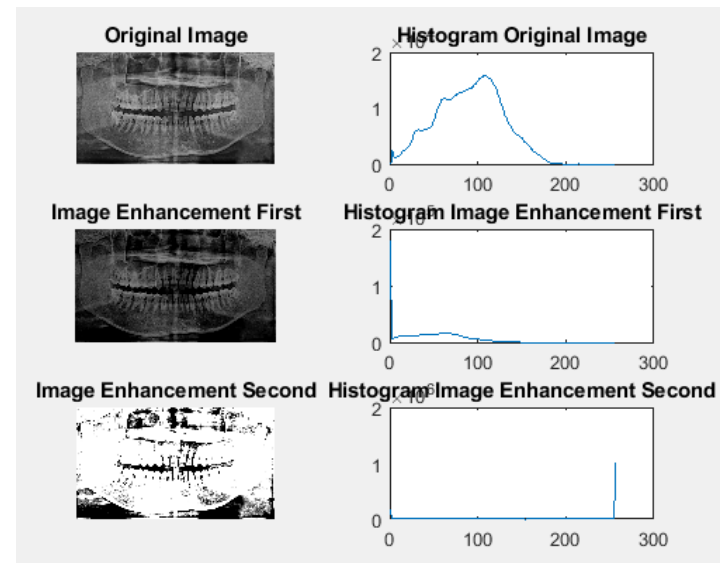

Fig. 6. Brightness Improvement and Contrast Stretch $\beta=-45, \alpha=11$

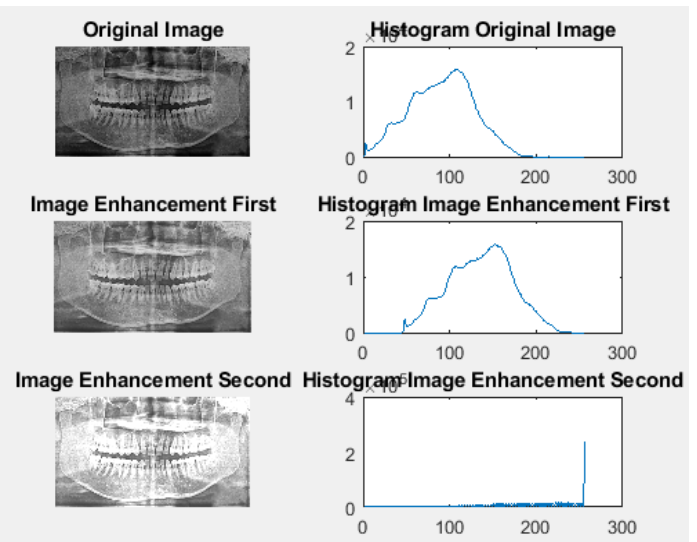

Fig. 7. Brightness Improvement and Contrast Stretch $\beta=45, \alpha=1.5$

\section{Active Contour Segmentation}

Active Contour is also called Snakes. Active Contours are contours that can change shape dynamically. Initial contours can be initialized randomly or based on user input. How to make an initialization with $v[0]$ and $v[1]$ as the start and endpoints of the contour. The way the Snake algorithm works is based on the minimum energy value, that is, the contour will move to find the minimum energy value as in Equation 4.

$$
J(v)=\int_{0}^{1} E(v(s)) d s
$$

$E(v(s))$ is the function evaluated at time s. The form of the energy function $E$ determines the characteristics of Snake. Energy function consists of two energies, namely internal energy $\left(E_{\text {int }}\right)$ and external energy $\left(E_{\text {ext }}\right)$, as in Equation 5.

$$
E(v(s))=E_{\text {int }}(v(s))+E_{\text {ext }}(v(s))
$$

Internal energy is energy from the Snake itself, and external energy is the energy that comes from the image itself. Internal energy is in charge of controlling changes in the contours. The internal energy is expressed in terms of the sum of the first and second derivatives as in Equation 6 .

$$
E(v(s))=\frac{1}{2}\left(\alpha\left\|\frac{\partial v(s)}{\partial s}\right\|^{2}+\beta\left\|\frac{\partial^{2} v(s)}{\partial s^{2}}\right\|^{2}\right)
$$

The larger the value, Snake will look like a straight line. And the greater the value, Snake is not flexible.
Values and must be adjusted appropriately so that the structure and shape are regular like Snake.

The external energy determines the direction Snake moves to reach the final position. The external energy comes from the image data itself. The external energy force can be written in Equation 7.

$$
\begin{aligned}
& E_{\text {ext }}^{1}(v(s))=|I(s)| \\
& E_{\text {ext }}^{2}(v(s))=-|\nabla I(s)|
\end{aligned}
$$

$E^{1}$ is the external energy derived from the intensity value of each image pixel, and $E^{2}$ is the negative value of the image gradient. If the user determines the initialization of the snake to a position far from the target, it can be overcome by making the image blurry. The image is blended using a Gaussian filter so that the external energy becomes as in Equation 8.

$$
\begin{aligned}
& E_{\text {ext }}^{3}(v(s))=\left|G_{\sigma}(s) * I(s)\right| \\
& E_{\text {ext }}^{4}(v(s))=-\left|G_{\sigma}(s) * \nabla I(s)\right|
\end{aligned}
$$
9

And merging with pixel gradient values like Equation

$$
E_{\text {ext }}^{5}(v(s))=w_{\text {line }}|I(s)|+w_{\text {edge }}|\nabla I(s)|
$$

\section{Postprocess}

Morphological operations are often performed on binary images. The purpose of this operation is to produce an image structure or image frame and object shape. This morphological operation implements the set operation. The set operation used is the logical AND or slice operation, and OR or a combination.

Several morphological operations include erosion, dilation, opening, and closing. Erosion is a morphological operation that aims to attenuate the image object or reduce the structure of the image object. The opening operation is a combination of erosion and dilation. The purpose of the opening operation is to smooth out the object and remove a small pixel area or to make the object image thinner.

\section{RESULTS AND DISCUSSION}

\begin{tabular}{|c|c|}
\hline TABLE I. CONTRAST CORRECTION TEST RESULTS \\
\hline $\begin{array}{c}\text { Original } \\
\text { image }\end{array}$ & Result \\
\hline $\begin{array}{c}\text { (Image- } \\
\text { average) } \\
x \\
\text { standard } \\
\text { deviation }\end{array}$ & \\
\hline
\end{tabular}

We did segmentation experiment with preprocess and post-process. The segmentation test used Active Contour as many as 86 panoramic radiographic images. We tested the preprocess with contrast enhancement of radiographic images with the following values:

a. $\beta=$ average image, and $\alpha=$ standard deviation, the results are as in Table I.

b. $\beta=$ image median, and $\alpha=$ image variance, the results are as in Table II. 
TABLE II. CONTRAST CORRECTION TEST RESULTS

\begin{tabular}{|c|c|}
\hline $\begin{array}{c}\text { Original } \\
\text { image }\end{array}$ & Result \\
\hline $\begin{array}{c}\text { Image } \\
\text { with } \\
\text { median } \\
\text { and } \\
\text { variance }\end{array}$ & \\
\hline
\end{tabular}

From the contrast improvement experiment in Table I and Table II, we exchanged the values of $\beta$ and $\alpha$, resulting in a contrast improvement as shown in Table III. Results that can be used for the next process are operations (image-average) $\times$ variance and (image-median) $\times$ standard deviation. However, both procedures have the same output, so we use the (image-average) $\times$ standard deviation.

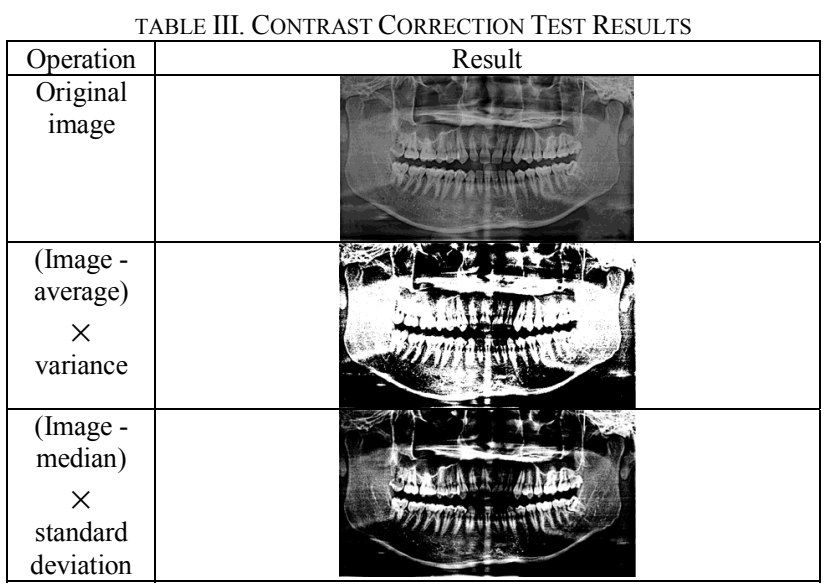

The Gaussian filter process aims to smooth the image or make the image blurry. We tested the Gaussian filter in three ways, as in Table IV. In Table IV, row 3 is the result of a Gaussian filter with a default sigma value of 2 (imgaussfilt (image repair, 2)), and in row 4 the sigma value is the standard deviation value of the image (imgaussfilt (repair_image, standard deviation)).

\begin{tabular}{|c|c|}
\hline \multicolumn{2}{c}{ TABLE IV. GAUSSIAN FILTER TEST RESULTS } \\
\hline Operation & \\
\hline $\begin{array}{c}\text { Original image } \\
\text { Gaussian }\end{array}$ & \\
\hline $\begin{array}{c}\text { Image with } \\
\text { Gaussian } \\
\text { sigma=2 }\end{array}$ & \\
\hline $\begin{array}{c}\text { Image with } \\
\text { Gaussian } \\
\text { sigma=standard } \\
\text { deviation }\end{array}$ & \\
\hline
\end{tabular}

In Table IV, we use the default sigma value of 2 and the sigma standard deviation of the image for the Active Contour process. A Gaussian filter with sigma $=2$ in Table IV shows that the results are better. So, we use a Gaussian filter sigma $=2$. After doing the contrast improvement preprocess and Gaussian filter, then segmenting it with Active Contour. The test results of the Active Contour are in Table V. Table $\mathrm{V}$ row 2 is the result of the Active Contour of the Gaussian filter image with sigma $=2$ and row 3 is the result of the Active Contour of the Gaussian filter image with the sigma value is the standard deviation of the image.

TABLE V. ACTIVE CONTOUR SEgMENTATION TeST RESUlts

\begin{tabular}{|c|c|}
\hline Operation & \\
\hline Original image & \\
\hline Ground Truth & \\
\hline $\begin{array}{c}\text { Active Contour } \\
\text { saussian filter }\end{array}$ & \\
\hline $\begin{array}{c}\text { Active Contour } \\
\text { Gaussian filter } \\
\text { sigma=standard } \\
\text { deviation }\end{array}$
\end{tabular}

TABLE VI. OPERATION MORPHOLOGY TEST RESUlTS

\begin{tabular}{|l|l|l}
\hline Operation & Result \\
\hline Active Contour & & \\
\hline Erosion & & \\
\hline
\end{tabular}

After segmenting with Active Contour, the next is post-process. Table V. We carried out the post-process erosion and opening morphology, the results of the erosion and opening morphology are shown in Table VI.

In Table VII, the level of similarity to ground truth is calculated. The process of calculating the level of similarity with ground truth uses Jaccard's similarity. The result of segmentation is a process carried out both before segmentation and after segmentation. The segmentation process first performs a preprocessing, then segmentation is carried out using Active Contour. The initial process by making improvements to the Contrast and Gaussian Filters. Active contours and post-processing were 
segmented using Active Contours, then erosion and opening morphology were performed. Preprocessing, Active Contour, and post-processing mean doing contrast enhancement and Gaussian filter then segmenting using Active Contour, and the final result is obtained by applying erosion and opening morphology.

We calculate the similarity value with Jaccard Similarity for all images. The Jaccard Similarity values are calculated between the segmentation results and the ground truth image. Table VII shows the average values of Jaccard Similarity from several methods and the highest average value achieve 0.52 by using Active Contour and Post-processing Opening method. The average value of similarity using Erosion method is 0.51 that comparable value with Opening method. The use of Post-processing outperforms to Pre-processing method. The highest Jaccard Similarity value achieves 0.67 also by using Active Contour and Post-processing Opening.

TABLE VII. JACCARD SIMILARITY RESULTS

\begin{tabular}{|l|c|}
\hline \multicolumn{1}{|c|}{ Methods } & $\begin{array}{c}\text { Average Jaccard } \\
\text { Similarity }\end{array}$ \\
\hline Active Contour & 0.5 \\
\hline Preprocessing + Active Contour & 0.34 \\
\hline $\begin{array}{l}\text { Preprocessing + Active Contour + Post- } \\
\text { processing (Erosion) }\end{array}$ & 0.31 \\
\hline $\begin{array}{l}\text { Preprocessing + Active Contour + Post- } \\
\text { processing (Opening) }\end{array}$ & 0.34 \\
\hline Active Contour + Post-processing (Erosion) & 0.51 \\
\hline $\begin{array}{l}\text { Active Contour + Post-processing } \\
\text { (Opening) }\end{array}$ & 0.52 \\
\hline
\end{tabular}

\section{CONCLUSION}

The results of Active Contour segmentation with postprocessing using opening morphology are better than other scenarios. The results of preprocessing segmentation (contrast improvement and Gaussian filter) and postprocessing (erosion and opening morphology) are compared. The result of the opening achieves a higher Jaccard similarity than the erosion in the post-processing morphological segmentation. The average Jaccard similarity of the Active Contour segmentation and postprocessing erosion is 0.51 . The average Jaccard similarity of the Active Contour and post-processing opening segmentation is 0.52 .

What can be done in the next research is to segment the mandible with more accurate results so that the mandible panoramic radiograph images are visible.

\section{ACKNOWLEDGMENT}

Thank you to the Indonesian Ministry of Research and Technology/National Research and Innovation Agency (RISTEK/BRIN) for providing research funding through the Doctoral Dissertation Research scheme with research contract number $1255 / \mathrm{PKS} / \mathrm{ITS} / 2020$. Thank you also to the Dental and Oral Hospital of Airlangga University, Surabaya for providing dental radiographic image data.

\section{REFERENCES}

[1] A. Ponnuswamy, Indira, A. Markande and M. P. David, "Mandibular Ramus: An Indicator for Sex Determination-A Digital Radiographic Study," Journal of Forensic Dental Sciences, pp. 5862, 2019.

[2] A. Damera, J. Mohanalakhsmi, P. K. Yellarthi and B. M. Rezwana, "Radiographic evaluation of mandibular ramus for gender estimation: Retrospective study," Journal of Forensic Dental Sciences, pp. 74-78, 2016.

[3] V. Sairam, G. R. Potturi, B. Praveen and G. Vikas, "Assessment of Effect of Age, Gender, and Dentoalveolar Changes on Mandibular Morphology: A Digital Panoramic Study," Clinical Dentisty, pp. 49-54, 2018.

[4] A. H. Abdi, S. Kasaei and M. Mehdizadeh, "Automatic Segmentation of Mandible in Panoramic X-ray," Journal of Medical Imaging, pp. 1-8, 2015.

[5] D.-J. Kroon, "Segmentation of the Mandibular Canal in ConeBeam CT Data," Universiteit Twente, Netherlands, 2011.

[6] R. Indraswari, T. Kurita, A. Z. Arifin, N. Suciati, E. R. Astuti and D. A. Navastara, "3D Region Merging for Segmentation of Teeth on Cone-Beam Computed Tomography Images," in International Conference on Soft Computing and Intelligent Systems and 19th International Symposium on Advanced Intelligent Systems, 2018.

[7] R. Indraswari, A. Z. Arifin, N. Suciati, E. R. Astuti and T. Kurita, "Automatic Segmentation of Mandibular Cortical Bone on ConeBeam CT Images Based on Histogram Thresholding and Polynomial Fitting," International Journal of Intelligent Engineering \& Systems, pp. 130-141, 2019.

[8] E. E. Nithila and S. Kumar, "Segmentation of lung from CT using various active contour models," Biomedical Signal Processing and Control, pp. 57-62, 2019

[9] A. B. Rabeh, F. Benzarti and H. Amiri, "Segmentation of Brain MRI using active contour Model," Wiley Periodicals, pp. 3-11, 2017.

[10] L. Fang, X. Wang and L. Wang, "Multi-modal medical image segmentation based on vector-valued active contour models," Information Sciences, pp. 1-15, 2019.

[11] A. Zareei and A. Karimi, "Liver segmentation with new supervised method to create initial curve for active contour," Computers in Biology and Medicine, pp. 139-150, 2016.

[12] L. Xu, Y. Zhu, Y. Zhang and H. Yung, "Liver segmentation based on region growing and level set active contour model with new signed pressure force function," Optik, pp. 1-11, 2019.

[13] C. Keatmanee, U. Chaumrattanakul, K. Kotani and S. S. Makhanov, "Initialization of active contours for segmentation of breast cancer via fusion of ultrasound, Droppler, and elasticity images," Ultrasonies, pp. 438-453, 2019.

[14] A. Ghosh and S. B. Yay, "Image co-segmentation using dual active contours," Applied Soft Computing, pp. 413-427, 2018.

[15] Z. Ying, L. Guangyao, S. Yiehua and Z. Yinmin, "Geometric active contours without re-initialization for image segmentation," Pattern Recognition, pp. 1970-1976, 2009.

[16] V. Caselles, R. Kimmel and G. Sapiro, "Geodesic Active Contours," International Journal of Computer Vision, pp. 61-79, 1997.

[17] J.-j. Zong, T.-s. Qiu, W.-d. Li and D.-m. Guo, "Automatic ultrasound image segmentation based on local entropy and active contour model," Computers and Mathematics with Applications, pp. 929-943, 2019.

[18] Riduwan, Skala Pengukuran Variabel Penelitian, Bandung: Alfabet, 2005

[19] E. Whaites and N. Drage, Essentials of Dental Radiography and Radiology, China: Elsevier, 2013.

[20] S. C. White and M. J. Pharoah, Oral Radiology Principles and Interpretation, China: Elsevier, 2014. 